\title{
La ola de los meta-análisis en red: una herramienta cada vez más útil para la toma de decisiones
}

Agustín Ciapponi ${ }^{\ddagger}$

Cortesía del Instituto de Efectividad Clínica y Sanitaria: iecs.org.ar

\begin{abstract}
Resumen
Cuando no disponemos de estudios que cotejen directamente las ventajas y desventajas de dos intervenciones, o cuando se necesita un ranking de las mejores intervenciones, este nuevo tipo de estudio ofrece una respuesta. En este artículo, el autor explica en qué consiste el meta-análisis en red y los requisitos que deben cumplir los estudios primarios para que sus resultados puedan ser combinados con esta metodología.
\end{abstract}

\section{Abstract}

When studies comparing risks and benefits of two interventions are not available, or when a ranking of the best interventions is needed, this new type of study offers an answer to clinical questions. In this article, the author explains what network metaanalysis is and what requirements primary studies must fulfill so that their results can be combined with this methodology

Ciapponi A. La ola de los meta-análisis en red: una herramienta cada vez más útil para la toma de decisiones. Evid Actual Pract Ambul. 2018;21(4):98-99

\section{Las tecnologías de la comunicación}

¿Cuál es el mejor tratamiento para detener crisis convulsivas? ¿Cuál es la terapia que mejor alivia el dolor en la enfermedad degenerativa vertebral? ¿Qué pacientes se benefician más de la terapia de mantenimiento de un cáncer de colon metastásico? ¿Los antifúngicos por vía oral son más efectivos que las soluciones o lacas tópicas para resolver los hongos de las uñas?

Tanto a nivel clínico como en la salud pública, las decisiones deben basarse en la mejor evidencia disponible; y aquellas relacionadas con intervenciones, idealmente, en revisiones sistemáticas y meta-análisis de ensayos clínicos aleatorizados (ECA). Sin embargo, con frecuencia se carece de evidencia directa y por cuestiones de costos o factibilidad, a menudo es altamente improbable que aparezca en el futuro ya que, ante avanzados tratamientos estándar, es cada vez más difícil demostrar beneficios adicionales.

Además, el meta-análisis tradicional o apareado (es decir, el que compara pares de intervenciones) tiene la desventaja de no permitir comparar múltiples alternativas terapéuticas al mismo tiempo, como sería necesario en las preguntas planteadas al comienzo de este artículo. Por otro lado, mucha evidencia proviene no de comparaciones realizadas contra un tratamiento estándar, sino contra placebo, lo que resulta menos útil para el médico o el tomador de decisiones que debe recomendar el tratamiento más adecuado.

Es en este escenario donde los meta-análisis en red (MAR), también llamados "meta-análisis con comparaciones múltiples" o "meta-análisis con comparaciones mixtas", aparecen como una nueva generación de estudios que incorporan las comparaciones indirectas junto al resto de la evidencia. De esta forma, se pueden obtener estimaciones de los efectos relativos de las diferentes alternativas terapéuticas a partir de comparaciones directas (si las hubiera) e indirectas, es decir, a partir de comparaciones mixtas.

Las agencias de evaluación de tecnologías, como el National Institute for Health and Care Excellence (NICE) en Reino Unido, el Canadian Agency for Drugs and Technologies in Health (CADTH), el Common Drug Review o el Pharmaceutical Benefits Advisory Committee (PBAC) en Australia y también Cochrane, estándar de calidad en el área de las revisiones sistemáticas y meta-análisis, consideran los resultados de los MAR como una fuente de información complementaria de importancia para guiar la toma de decisiones en distintos niveles. De hecho, su número ha crecido de manera vertiginosa, en especial, desde 2009. La tendencia creciente de la publicación de MAR ya la habíamos apreciado en las principales cuatro bases de datos ${ }^{1}$ y continuó en ascenso. Por ejemplo, en 2010, la literatura científica indizada en PubMed registraba 23 artículos que referían a ese tipo de estudios, pero la cifra trepó a 257 en 2014 y 665 en 2017.

Figura 1. Representación esquemática de las posibles comparaciones entre intervenciones en el meta-análisis en red.

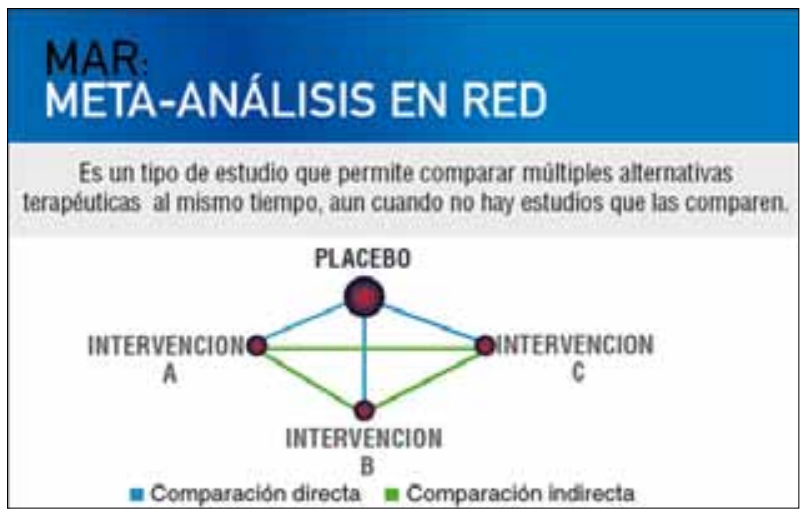

\section{Diseño y requisitos}

Si estuviéramos interesados en la eficacia o eficiencia comparativa entre la intervención $A$ versus $B$, por los métodos tradicionales sólo podríamos estimarla si han sido comparados directamente. Sin embargo, podríamos obtener evidencia indirecta inferida de ensayos aleatorizados de A versus $C$ y $B$ versus $C$ (ver fig.1). Esto es una simplificación, pues las redes suelen tener mucho más de tres nodos (intervenciones) y tienen la virtud de poder nutrirse de cada aporte para mejorar la precisión de las estimaciones. Sin embargo, las comparaciones indirectas son más débiles que las directas si no sabemos qué tan comparables son los estudios entre sí.

Es por eso que la validez de las comparaciones indirectas va a depender primariamente, además de la calidad metodológica de los estudios considerados, de la variabilidad entre estudios. Un primer requisito es que los estudios a incluir en un MAR sean razonablemente homogéneos. De hecho, la homogeneidad (o ausencia de heterogeneidad) de resultados es requerida a todo meta-análisis.

Adicionalmente, los MAR basan sus estimaciones en dos asunciones o supuestos específicos: la transitividad (similitud) y la coherencia (consistencia) $)^{2-4}$.

El término transitividad se refiere a que asumimos que podemos comparar dos tratamientos a través de un tercero en común. El

‡ Director Cochrane Argentina y Centro Cochrane Argentino IECS. 
investigador debe verificar que diferentes estudios incluidos en un MAR sean comparables por no diferir en la distribución de factores modificadores de efecto como el diseño de los estudios, gravedad de los pacientes, tratamientos concomitantes, etc. Una forma práctica de chequear la asunción de transitividad es respondiendo afirmativamente la pregunta: ¿Podrían los pacientes incluidos en un MAR ser aleatorizados a cualquiera de los tratamientos de la red?

La otra gran asunción es la coherencia o "consistencia", que expresa el grado de acuerdo entre la evidencia directa e indirecta, lo cual es fundamental para decidir combinar o no esa evidencia mixta dentro de un MAR. La coherencia sólo puede evaluarse para todas las comparaciones que cuentan con ambas estimaciones de efecto (directa e indirecta).

Es importante notar que la metodología del MAR no se limita a la estimación numérica de diversas comparaciones indirectas o mixtas, sino que también permite explorar sesgos y proponer una jerarquía o ranking de intervenciones de acuerdo con la probabilidad de ser el mejor tratamiento. Sin embargo, hay que utilizarlos con cautela porque pueden ser engañosos. Por ejemplo, la intervención que encabeza el ranking de eficacia no necesariamente es la que se basa en la mejor calidad de la evidencia. En este sentido, el método GRADE permite evaluar la evidencia provista por los MAR.

Comúnmente, los MAR se representan gráficamente como una red $^{2-4}$. En la figura 2 se presentan las múltiples comparaciones directas y la red completa de evidencia para la eficacia de diferentes tratamientos orientados a prevenir caries dentales a partir de la información procedente de una revisión sistemática. Para representar cada tratamiento, se pueden utilizar nodos (cada vértice representa las intervenciones que compiten) $\mathrm{y}$, cuando existen estudios que presentan comparaciones directas, se establecen líneas o conexiones entre ellos. El grosor de la línea expresa la cantidad de estudios existentes con cada comparación directa y el color puede representar el riesgo de sesgo.
Por otra parte, su metodología también evoluciona de manera acelerada, incluyendo la combinación de evidencia proveniente de estudios aleatorizados y no aleatorizados ${ }^{5}$, así como la incorporación de nueva evidencia en tiempo real (MAR "vivos") ${ }^{6}$.

En síntesis, los MAR son un tipo de estudio de investigación que, teniendo en cuenta la totalidad de la evidencia disponible para una condición dada, cubre el espectro de todas las intervenciones de interés e incorporan comparaciones tanto directas como indirectas para establecer los efectos y mostrarlos de forma panorámica.

Los MAR presentan ventajas y desventajas respecto de los metaanálisis tradicionales. Pero cuando no se cuenta con evidencia directa y/o hay múltiples alternativas terapéuticas, resultan verdaderamente irremplazables.

Figura 2. Representación gráfica de la red de comparaciones directas e indirectas en un meta-análisis de intervenciones para prevenir las caries dentales.

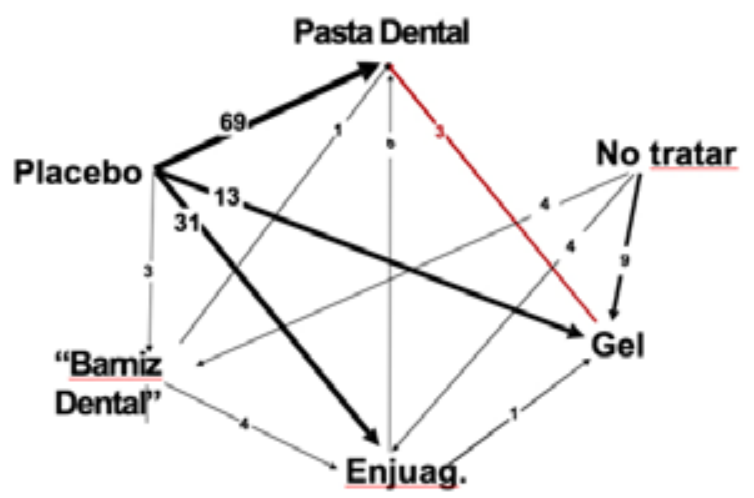

Tomado de: Caldwell D, Basic ideas of indirect comparisons and network meta-analysis. Cochrane. Comparing Multiple Interventions Methods Group Oxford Training event, 2013

Referencias

1. Ciapponi A y col. The growing trend of indirect comparisons meta-analyses topic appearance in medical literature. Paper presented at: XXI Cochrane Colloquium. Better Knowledge for Better Health; 19-23 September 2013, 2013; Quebec, Canada.

2. Brignardello-Petersen $\mathrm{R}$ y col. Advances in the GRADE approach to rate the certainty in estimates from a network meta-analysis. J Clin Epidemiol. 2018;93:36-44.

3. Puhan MA y col. A GRADE Working Group approach for rating the quality of treatment effect estimates from network meta-analysis. BMJ. 2014;349:g5630.

4. Salanti $G$ y col. Evaluating the quality of evidence from a network meta-analysis. PLoS One. 2014;9(7):e99682.

5. Efthimiou $O$ y col. Combining randomized and non-randomized evidence in network meta-analysis. Stat Med. 2017.

6. Nikolakopoulou A y col. Living network meta-analysis compared with pairwise meta-analysis in comparative effectiveness research: empirical study. BMJ. $2018 ; 360: k 585$.

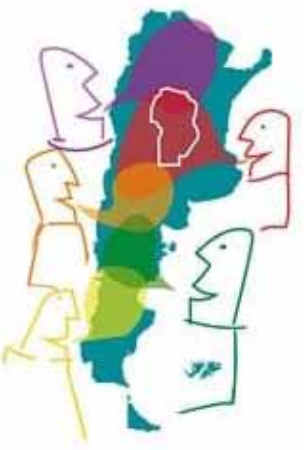

\title{
XVIII Congreso Nacional de MEDICINA FAMILIAR Y GENERAL FAMFYG
}

\author{
- XVI Congreso Provincial Acomfyg \\ - XVIII Jornadas Nacionales de Residentes \\ - VI Congreso de Profesores de Medicina Familiar
}

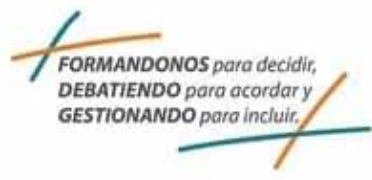

